\title{
Study of the turbulent swirl flow in the pipe behind the axial fan impeller
}

\author{
Đorde S. Čantrak ${ }^{1, a}$, Vesna Mila Z. Čolić Damjanović ${ }^{2}$ and Novica Z. Janković ${ }^{1}$ \\ 1 University of Belgrade, Faculty of Mechanical Engineering, Belgrade, Serbia \\ ${ }^{2}$ University of Belgrade, Faculty of Architecture, Belgrade, Serbia
}

Received 27 May 2015, Accepted 29 February 2016

\begin{abstract}
Experimental research of the turbulent swirl flow in the pipe behind the axial fan impeller is presented in this paper. Axial fan is inbuilt without guide vanes in the installation with a free inlet and ducted outlet, like it is widely used in the ventilation systems. One-component laser Doppler anemometry and stereo particle image velocimetry (SPIV) are used in two measuring sections on the fan pressure side and for three fan rotation numbers. Non-dimensional axial velocity profile is not significantly transformed downstream, while the circumferential is, although it preserves its character. Turbulence level is the highest in the vortex core in both measuring sections for all velocities. It increases downstream in the vortex core zone and decreases in the main flow region. Determined skewness and flatness factors point out the intermittent character of the generated turbulent swirl flow, as well as the existence of the organized coherent structure. Correlation curves indicate various dynamics of fluctuating circumferential velocity fields in measuring points. The Rankine vortex structure of the turbulent swirl flow is also revealed by the SPIV measurements. Analysis shows more dominant vortex core dynamics in the downstream section. Studied flow is characterized by extensive mass, momentum and energy transfer.
\end{abstract}

Key words: Axial fan / turbulent swirl flow / PIV / LDA

\section{Introduction}

Turbulent swirl flow occurs in numerous technical systems, as well as in nature. They are present in turbomachinery, cyclones, combustion processes, magneto-hydrodynamic generators, inducers etc. A vortex structure at the inlet of an inducer is studied in reference [1], while mean and turbulent features of inducer inlet flow for various centrifugal pump duty points are analyzed in reference [2]. Paper's focus is on the turbulent swirl flow generated by the axial fan in a straight pipe, where it can be studied as the swirl flow generator, i.e., inducer. Ducting adjacent to an axial fan have a considerable effect on the fan duty point. It is shown that a fan will achieve its optimum performance when the flow at the inlet is fully developed with symmetrical air velocity profile which is free from swirl. Similar situation is necessary at the fan discharge [3]. It is also reported in reference [3], page 102: "In the case of tube axial fans, the problem can be especially severe with swirl existing up to almost 100 diameters of ducting. The only solution is to incorporate a flow straightener, which destroys the swirl,

a Corresponding author: djcantrak@mas.bg.ac.rs or guide vanes which can recover the swirl energy". This is also shown in reference [4].

This paper presents a study of the turbulent swirl flow on the axial fan pressure side by the use of the laserbased, measuring techniques such as particle image velocimetry (PIV) and laser Doppler anemometry (LDA) in the ventilation ducts.

The paper's focus is on the turbulent swirl flow generated by the axial fan in a straight pipe. It is inbuilt without guide vanes in the installation with a free inlet and ducted outlet categorized as B installation type in ISO 5801. It is widely used in ventilation systems in this way. This case is studied theoretically, experimentally and numerically, not exclusively, but thoroughly in references [4-10]. Installation of the C category after ISO 5801 has been recently studied in reference [11]. The development of the mean velocity field of the turbulent swirl flow in straight pipes is described with a thorough overview in reference [12]. Complex analysis of turbulent swirl flow, which is of interest in HVAC systems, is given in reference [13]. Some aspects of application of the laser-based measurement and visualization techniques in ventilation systems are reported, not only in reference [6]. 


\section{Nomenclature}

\begin{tabular}{|c|c|}
\hline$c$ & Velocity magnitude, $\mathrm{m} \cdot \mathrm{s}^{-1}$ \\
\hline$D$ & Inner pipe diameter, $\mathrm{m}$ \\
\hline$F$ & Flatness factor \\
\hline$n$ & Fan shaft rotational speed, rpm \\
\hline$Q$ & Volume flow rate, $\mathrm{m}^{3} \cdot \mathrm{s}^{-1}$ \\
\hline$R$ & Inner pipe radius, $m$ \\
\hline$r$ & Radial coordinate, $\mathrm{m}$ \\
\hline$R e$ & Reynolds number \\
\hline$S$ & Skewness factor \\
\hline$U$ & Mean axial velocity, m.s $\mathrm{s}^{-1}$ \\
\hline$u$ & Fluctuating axial velocity, $\mathrm{m} . \mathrm{s}^{-1}$ \\
\hline$V$ & Mean radial velocity, $\mathrm{m} \cdot \mathrm{s}^{-1}$ \\
\hline$v$ & Fluctuating radial velocity, $\mathrm{m} . \mathrm{s}^{-1}$ \\
\hline$W$ & Mean circumferential velocity, m.s ${ }^{-1}$ \\
\hline$w$ & Fluctuating circumferential velocity, $\mathrm{m} . \mathrm{s}^{-1}$ \\
\hline$x, y$ & Target coordinate system, mm \\
\hline$z$ & Axial coordinate along a pipe axis, $\mathrm{m}$ \\
\hline \multicolumn{2}{|r|}{ Greek symbols } \\
\hline$\alpha$ & Dimensionless radius \\
\hline$\beta_{R}$ & Blade angle at impeller diameter, ${ }^{\circ}$ \\
\hline$\Gamma$ & Average circulation, $\mathrm{m}^{2} \cdot \mathrm{s}^{-1}$ \\
\hline$\nu$ & Kinematic viscosity, $\mathrm{m}^{2} \cdot \mathrm{s}^{-1}$ \\
\hline$\sigma$ & $\begin{array}{l}\text { Root-mean-square of the turbulent velocity } \\
\text { fluctuations, } \mathrm{m} . \mathrm{s}^{-1}\end{array}$ \\
\hline$\tau$ & Correlation time, s \\
\hline$\varphi$ & $\begin{array}{l}\text { Coordinate of the polar cylindrical } \\
\text { coordinate system, }^{\circ}\end{array}$ \\
\hline$\Omega$ & Swirl flow parameter \\
\hline
\end{tabular}

Experimentally obtained and presented results in this paper show the turbulent swirl flow durability. Namely, the turbulent swirl flow with the Rankine vortex structure, generated by the specially designed axial fan, survived till the downstream measuring section, which is almost at the test rig outlet. Velocity profile has been changed, but not significantly. Experimentally determined skewness and flatness factors reveal the intermittent character of the generated turbulent swirl flow and existence of the organized coherent structure. In addition, the calculated correlation curves indicate various dynamics of the fluctuating circumferential velocity fields in various flow regions.

Vortex core dynamics is documented in this paper by the PIV study and analysis. It is shown that the vortex core dynamics is greater in the downstream measuring section. This is of great importance for the ventilation systems design.

\section{Experimental test rig}

Experimental test rig is presented in Figure 1. The first measuring section is positioned in $z / D=3.35$, while the second in $z / D=26.31$, where $z$ is the axial coordinate measured from the test rig inlet, i.e., the plane tangential to the profiled inlet and $D=0.4 \mathrm{~m}$ is an average inner diameter. Total experimental test rig length is $27.74 \mathrm{D}$.

The axial fan impeller is designed to operate with guide vanes, but here it is employed without them. This is still very common in technical practice. It has nine blades, the outer diameter is $D_{a}=0.399 \mathrm{~m}$ (Fig. 2a) and the dimensionless diameter, which is the ratio of the hub and the outer diameter, is $\alpha=0.5$. NACA 0010 profiles are used. The blade pitch angle is positioned at $\beta_{R}=30^{\circ}$. The axial fan is inbuilt with a free inlet and ducted outlet, specified as category B in the international standard ISO 5801:2007. Flow at the inlet is fully developed with a symmetrical air velocity profile and it is free of swirl. This is checked by use of the smoke visualization technique. Its length, with attached hydraulically profiled cap, is $295.5 \mathrm{~mm}$ (Fig. 2b). It is designed to generate the Rankine vortex, i.e., the circumferential velocity distribution $r W=$ const., where $r$ is a radius and $W$ is time averaged circumferential velocity. Fan inbuilt characteristics are defined in Figure 2b. Inlet cap is a part of the fan hub (Fig. 2b pos. 1) and has equation $y=\left(a x^{2}+b x+c\right) /\left(x^{3}+d x^{2}+e x+f\right)$, where $x$ is axis along the pipe axis, $a, b, c, d, e$ and $f$ are constants. Profiled cap at the fan discharge side (pos. 2 at Fig. 2b) is defined with two radii: R52 with center at the pipe axis and $108 \mathrm{~mm}$ from the cap bottom and R200 with the center on the cap basis diameter.

The fan rotation speed was regulated by a fully automated thyristor bridge with an error up to $\pm 0.5 \mathrm{rpm}$. Measurements were performed on the characteristic axial fan impeller rotation number $n=1000,1500$ and $2000 \mathrm{rpm}$, which generated the extensively turbulent swirl flow.

\section{Measurement techniques}

The study of the turbulent swirl flow employed laser-based measuring techniques, such as laser Doppler anemometry (LDA) and stereo particle image velocimetry (SPIV). Stereo PIV and LDA measurements were performed on the axial fan pressure side in the specified measuring sections (positions 4 and 5 in Fig. 1).

\subsection{Laser Doppler anemometry (LDA)}

The LDA measurements were performed subsequently for all three velocity components using a one-component LDA system along the vertical diameter at the points, at a 10-mm distance each, in specified sections (Fig. 3). A half plane above the pipe axis is denoted as $\phi=90^{\circ}$, while under the pipe the axis is $\phi=270^{\circ}$ (Fig. 5a). The laser was mounted on the computer controlled linear guides. The system works in a backscattered mode. The LDA system is a Flow Explorer Mini LDA, Dantec, with signal processors BSA F30. Light wavelength is $660 \mathrm{~nm}$ and the optics used had a focus $300 \mathrm{~mm}$. Measuring uncertainty of the used LDA system, after manufacturer's specification, is $0.1 \%$ for the whole system. In addition, a brief overview 


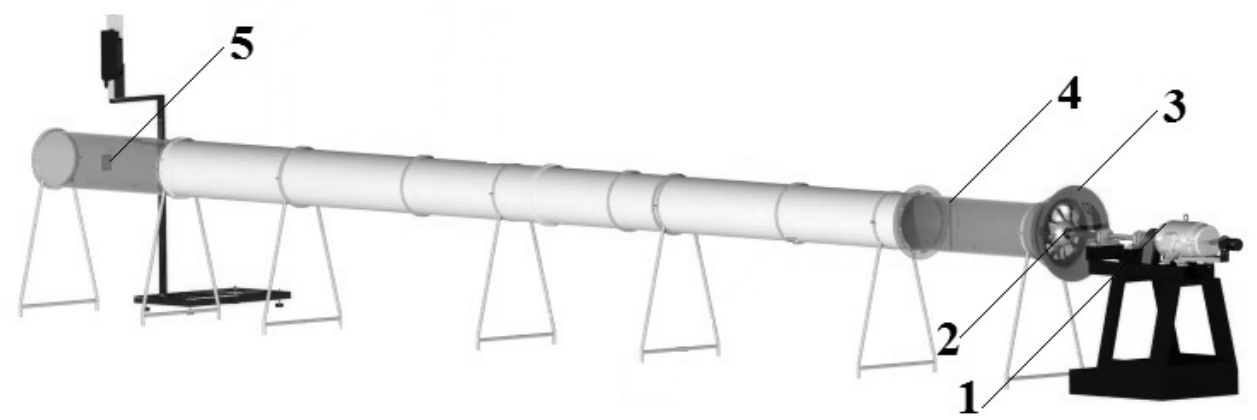

Fig. 1. Experimental test rig: 1 - DC motor, 2 - axial fan impeller, 3 - profiled free bell-mouth inlet, 4 - first measuring section for LDA and stereo PIV measurements $(z / D=3.35)$ and 5 - second measuring section for LDA and stereo PIV measurements $(z / D=26.31)$.

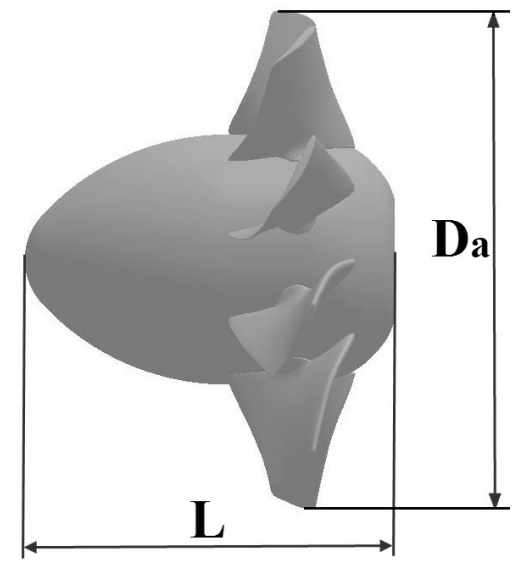

(a)

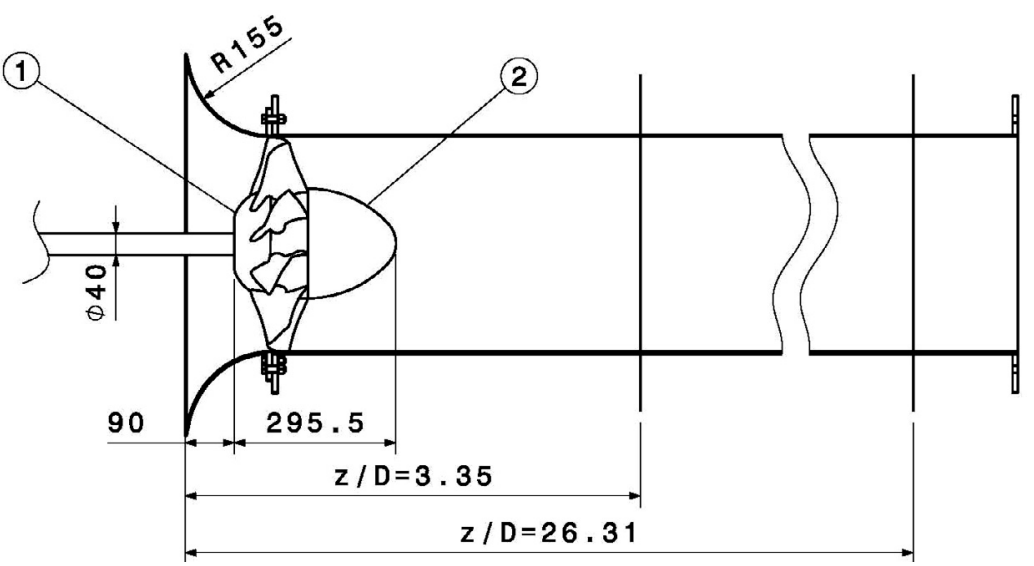

(b)

Fig. 2. Axial fan impeller: (a) dimensions in the meridian plane and (b) inbuilt dimensions.

of uncertainty sources in the LDA measurements is reported in reference [14]. Estimation of LDA measuring volume positioning in a cylindrical pipe due to optical aberrations caused by the pipe wall curvature is studied in references $[14,15]$. The transit time was used as the weighting factor. Recording time of $10 \mathrm{~s}$ was set up as a stop criterion for all measurements. Data frequency varied along the vertical diameter, depending of the measured velocity component. Data validation during the test was, on average, $85 \%$. Sensitivity was adjusted to the values 1200-1400 V. The acquisition and part of data processing were done in the BSA software, while the major part of data processing was done by the self-made programs. The flow was seeded by an Antari Z3000II fog machine with liquid "Heavy fog". It was naturally sucked in the test rig by the fan and consequently enough seeding was obtained.

\subsection{Stereo particle image velocimetry (SPIV)}

Figure 3b displays the stereo PIV calibration in the first measuring cross-section, i.e., $z / D=3.35$. Stereo PIV measurements were performed in the cross-section and vertical meridian section with origins in specified measuring sections.

The target coordinate systems, defined as in the Insight 3G software, are presented in Figure 4, which corresponds to Figure 3 a.

The laser and cameras form a back scatter Scheimpflug setup. Dual head Nd:YAG laser (30 mJ/pulse, wavelength $532 \mathrm{~nm}$, and $15 \mathrm{~Hz}$ ) illuminated the flow. Two 12-bit CCD cameras with the resolution of $1660 \times 1200$ pixels and $32 \mathrm{fps}$ were used. Data acquisition and raw images data processing were performed by the Insight 3G software, while postprocessed in Tecplot. Two data sampling modes were used, first with $2 \mathrm{~Hz}$, when 400 pictures were collected, and the second one with $7 \mathrm{~Hz}$, when only 99 raw images were collected. Raw images processing was performed using the CDIC (central difference image correction) deformation algorithm in combination with FFT correlator [16]. This is a four-pass method and it employed a square interrogation area of $32 \mathrm{px}$. Velocity vectors were validated using the velocity range criteria and $3 \times 3$ local median filter, while missing vectors were interpolated using a $3 \times 3$ local mean technique. The same seeding was used as in the LDA measurements. 


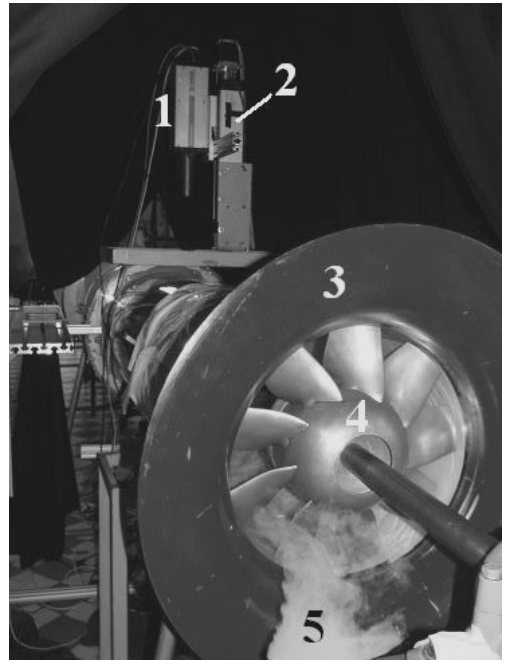

(a)

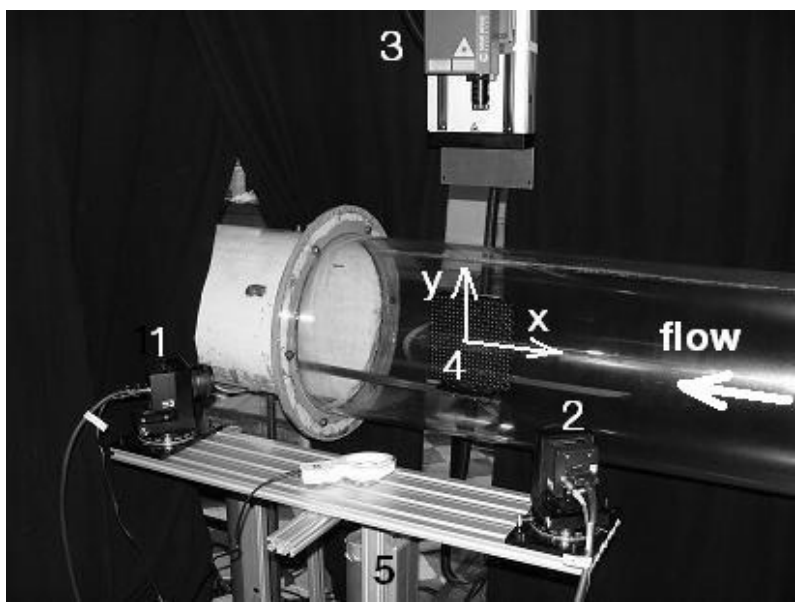

(b)

Fig. 3. Measurements in section $z / D=3.35$ : (a) One-component LDA measurements: circumferential velocity in measuring section: 1 - LDA laser, 2 - linear guide, 3 - profile inlet, 4 - axial fan and 5 - seeding; (b) Stereo PIV system calibration in the first cross-section: 1 - left and 2 - right CCD camera, 3 - Nd:Yag laser, 4 - target and 5 - linear guide.

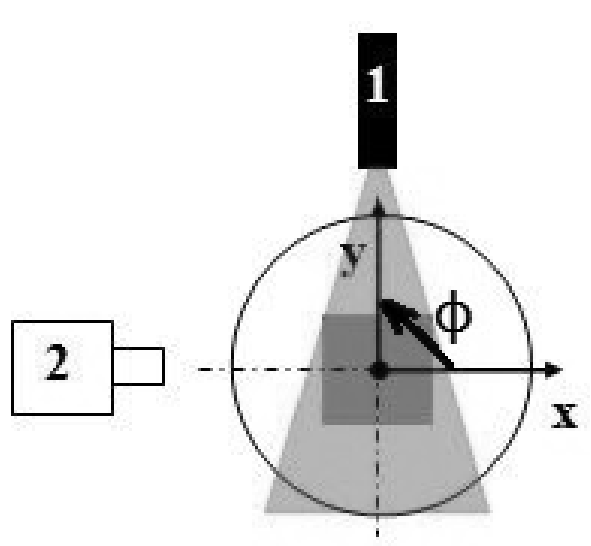

(a)

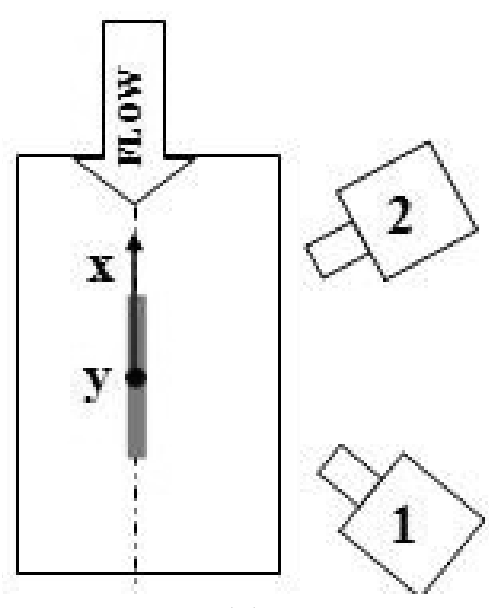

(b)

Fig. 4. Target coordinate systems definition for measurements in: (a) cross-section (view from the fan side): 1 - Nd:YAG laser and 2 - cameras and (b) vertical meridian section (view from above): 1 - left and 2 - right camera.

\section{Experimental results and analysis}

\subsection{LDA measurements}

\subsubsection{Integral characteristics}

Experimentally obtained distributions of time averaged axial and circumferential velocity fields for all three regimes, generated by the axial fan impeller, in the measuring section $z / D=3.35$ are presented in Figure 5 .

It is obvious that the generated circumferential velocity profile $(W)$ is similar to the model of the solid bodypotential swirl, i.e., the Rankine vortex. In the central zone, the turbulent vortex core is formed, known as the forced vortex region, with the solid body distribution of circumferential velocity, where $W \propto r$. This domain, with a shear layer characterized by $W \approx W_{\max }$, is connected to the sound (or main) flow region or free vortex, where $U \approx$ const, and circumferential velocity has the distribution of the potential swirl $W \propto 1 / r$. The fourth - the wall region is characterized by the properties of flow in a boundary layer, which is not quantified in these experiments. A reverse flow exists in both measurement sections (Fig. 5a). It is obvious that the character of the curves is similar for all three regimes in all four flow regions.

On the basis of experimentally obtained distributions of time averaged axial $(U)$ and circumferential $(W)$ (Fig. 5) velocities obtained by the one-component LDA in the measuring section $z / D=3.35$, the flow integral characteristics were calculated: the volume flow rate $(Q)$, the average velocity in volume $\left(U_{m}=Q /\left(R^{2} \pi\right)\right)$, 


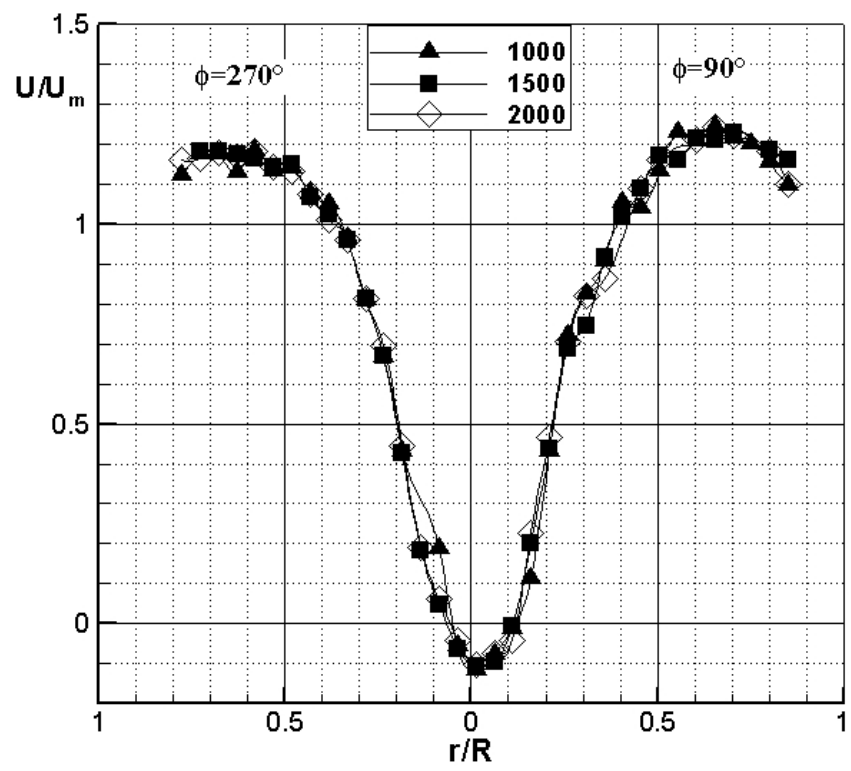

(a)

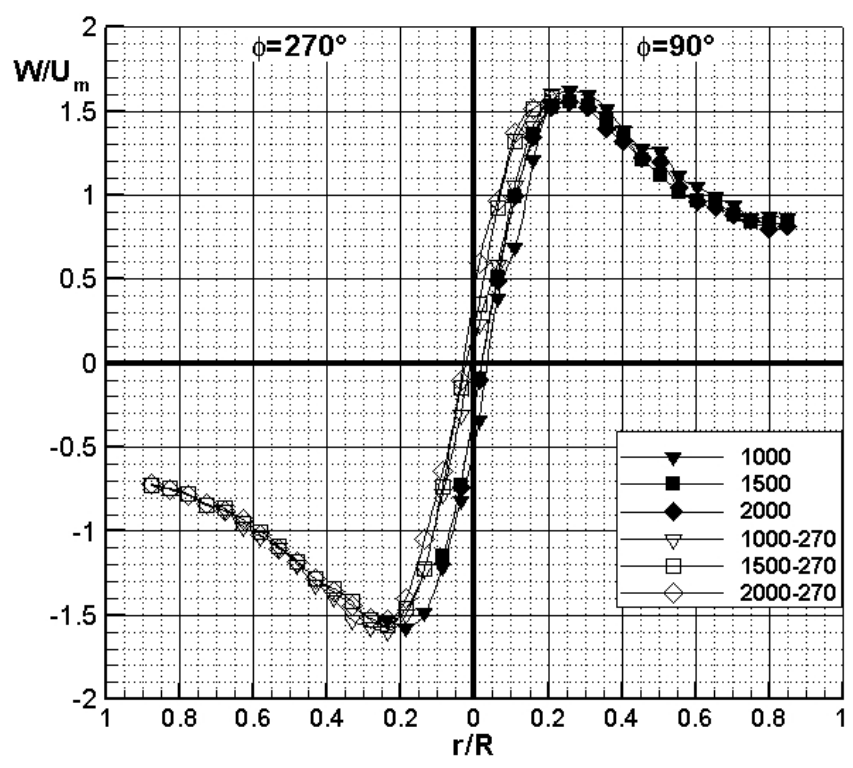

(b)

Fig. 5. Radial distribution of time averaged velocities in measuring section $z / D=3.35$ for all three regimes $n=1000$, 1500 and $2000 \mathrm{rpm}$ : (a) axial velocity $(U)$ and (b) circumferential velocity $(W)$.

Table 1. Calculated integral parameters in the measuring section $z / D=3.35$.

\begin{tabular}{cccccc}
\hline \multirow{2}{*}{ Regime $[\mathrm{rpm}]$} & \multicolumn{5}{c}{ Integral parameters } \\
\cline { 2 - 6 } & $Q\left[\mathrm{~m}^{3} \cdot \mathrm{s}^{-1}\right]$ & $U_{m}\left[\mathrm{~m}_{\mathrm{s}}^{-1}\right]$ & $R e$ & $\Gamma\left[\mathrm{m}^{2} \cdot \mathrm{s}^{-1}\right]$ & $\Omega$ \\
\hline 1000 & 0.86 & 6.68 & 182602 & 5.41 & 0.79 \\
1500 & 1.305 & 10.13 & 277018 & 7.91 & 0.81 \\
2000 & 1.741 & 13.52 & 369612 & 10.51 & 0.82 \\
\hline
\end{tabular}

the Reynolds number (Re $=U_{m} D / \nu$, where $\nu$ is kinematic viscosity), the average circulation $(\Gamma=$ $\left.4 \pi^{2} R^{3} \int_{0}^{1} U W(r / R)^{2} \mathrm{~d}(r / R) / Q\right)$ and the swirl number ( $\Omega=Q /(R \Gamma)$, where $R$ is the inner pipe radius in this cross-section). Calculated integral parameters for the measuring section $z / D=3.35$ are given in Table 1 .

Averaged velocity fields evolution is shown with radial-axial distributions in Figure 6 for one regime $n=$ $1500 \mathrm{rpm}$. Circumferential velocity strongly influences the distributions of axial $(U)$ and radial $(V)$ velocities. It is obvious that the downstream transformation of the circumferential velocity is more intensive than the axial velocity (Fig. 6). Circumferential velocity $W$ decreases downstream in all points of the cross-section, but preserves the character of the Rankine vortex (Fig. 6b). The reverse flow region is preserved for the regime $n=$ $1500 \mathrm{rpm}$, also in the downstream region.

Calculated integral parameters for the section $z / D=$ 26.31 and regime $n=1500 \mathrm{rpm}$ are: $Q=1.27 \mathrm{~m}^{3} \cdot \mathrm{s}^{-1}$, $U_{m}=10.11 \mathrm{~m} \cdot \mathrm{s}^{-1}, R e=275344, \Gamma=5.4 \mathrm{~m}^{2} \cdot \mathrm{s}^{-1}$ and $\Omega=1.17$. Relative difference in the measuring sections $z / D=3.35$ and $z / D=26.31$ between the obtained volume flow rates for the same regime, defined by the axial impeller rotation number $n=1500 \mathrm{rpm}$, is $2.68 \%$. It is shown that the swirl flow decays downstream, as expected.
Downstream transformation of the radial velocity for the same regime ( $n=1500 \mathrm{rpm})$ is shown in Figure 6c. It has higher values in the main flow region in the section $z / D=3.35$ than in the section $z / D=26.31$, while the opposite situation is in the vortex core region.

\subsubsection{Turbulence statistics}

Figure 7 shows the turbulence levels for all three regimes for circumferential velocity $\left(\sigma_{w} / U_{m}\right)$ in the measuring section $z / D=3.35$. Here, the study involved the influence of the fan rotation number on the statistical characteristics of the generated field of the circumferential fluctuating velocities due to the dominant role of the circumferential velocity. Studied parameters of this influence are the turbulence level, skewness and flatness factors.

Turbulence intensities in axial $\left(\sigma_{u}\right)$, radial $\left(\sigma_{v}\right)$ and circumferential $\left(\sigma_{w}\right)$ directions are defined after the following expressions:

$$
\begin{aligned}
& \sigma_{u}=\left(\overline{u^{2}}\right)^{1 / 2}=\left[\frac{1}{T} \int_{0}^{T} u^{2} \mathrm{~d} t\right]^{1 / 2}, \sigma_{v}=\left(\overline{v^{2}}\right)^{1 / 2}, \\
& \sigma_{w}=\left(\overline{w^{2}}\right)^{1 / 2},
\end{aligned}
$$




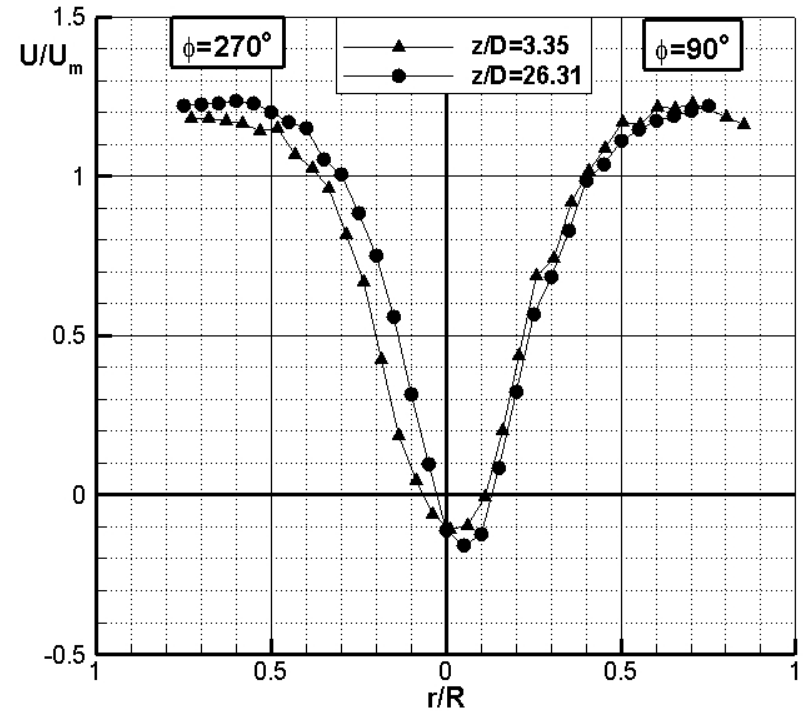

(a)

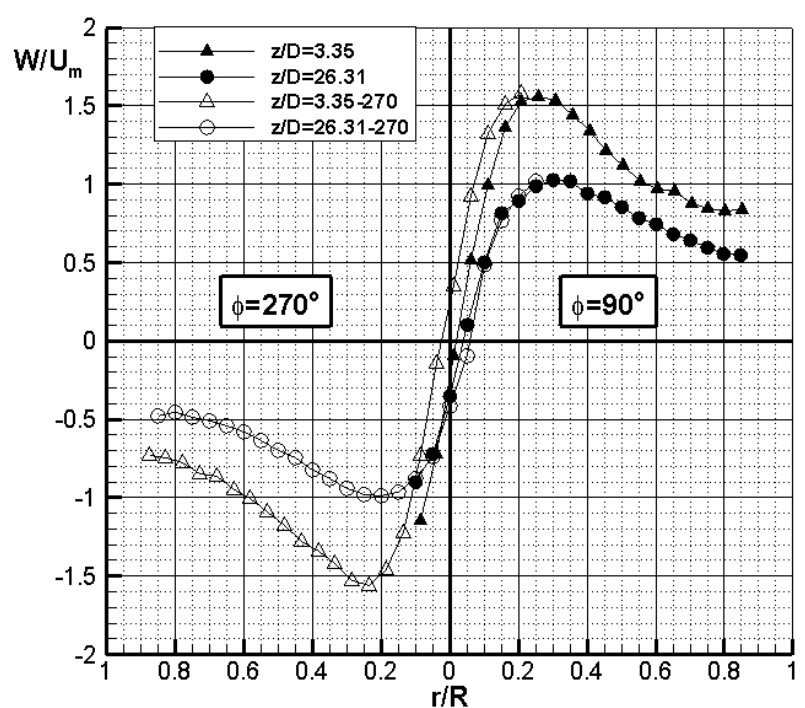

(b)

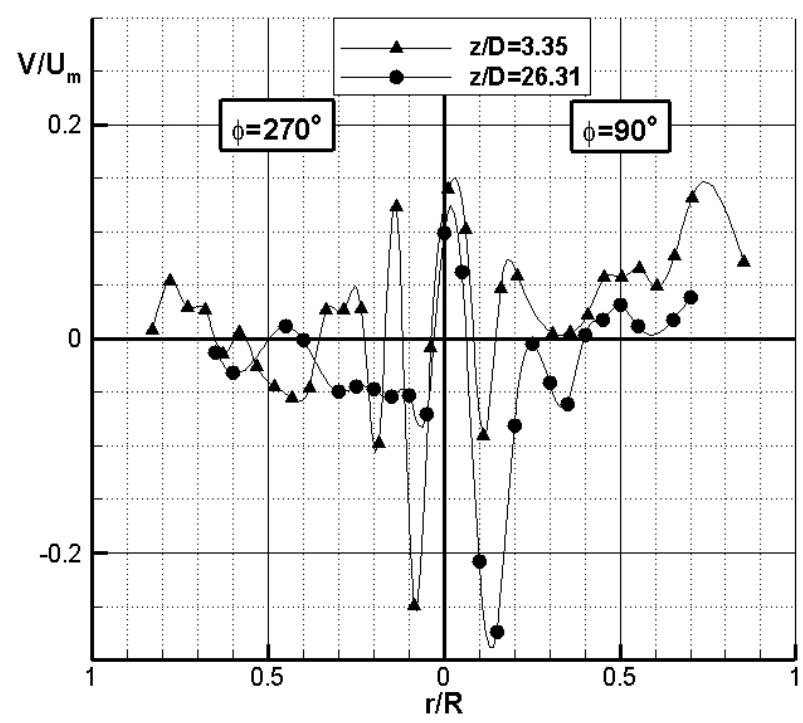

(c)

Fig. 6. Radial distribution of time averaged velocities in measuring sections $z / D=3 / 35$ and $z / D=26.31$ : (a) axial $(U)$, (b) circumferential $(W)$ and (c) radial $(V)$ velocity.

where $u_{i}=u, v, w$ are axial, radial and circumferential velocity fluctuations. The values of the normalized central moments for all three velocity components of the third $S_{i}$ (skewness), and the fourth order $F_{i}$ (flatness) are calculated as follows:

$$
S_{i}=\overline{u_{i}^{3}} / \sigma_{i}^{3}, F_{i}=\overline{u_{i}^{4}} / \sigma_{i}^{3} .
$$

For normal, i.e., Gaussian statistical distributions, the skewness factor has the value 0 , while the flatness factor equals $3\left(S_{i}=0\right.$ and $\left.F_{i}=3\right)$.

The highest sampling rates are achieved for circumferential velocity. The character of the turbulence level $\left(\sigma_{w} / U_{m}\right)$ distributions is similar for all regimes. The highest turbulence levels, for all regimes, are achieved in the region up to $r / R=0.2$. Namely, $\left(\sigma_{w} / U_{m}\right)_{\max } \approx 0.28$ is achieved for the highest rotation number $n=2000 \mathrm{rpm}$ (Fig. 7a). In this region the highest turbulence level gradients are also achieved. More uniform distributions occur in the sound flow region. The sign of the skewness factor for circumferential velocity $\left(S_{w}\right)$ determines the directions of the turbulent diffusion. $S_{w}$ is not equal to zero and changes its sign for all three regimes.

Figure 8 shows the downstream evolutions of distributions of the turbulence level $\left(\sigma_{w} / U_{m}\right)$ as well as normalized correlation moments of the third and fourth order for fluctuating velocities in the circumferential direction $S_{w}$ and $F_{w}$ for the regime $n=1500 \mathrm{rpm}$. The circumferential velocity downstream transformation, its influence on the other two components, and the highest sampling rates (in some cases higher than $30 \mathrm{kHz}$ ) were the main reasons 


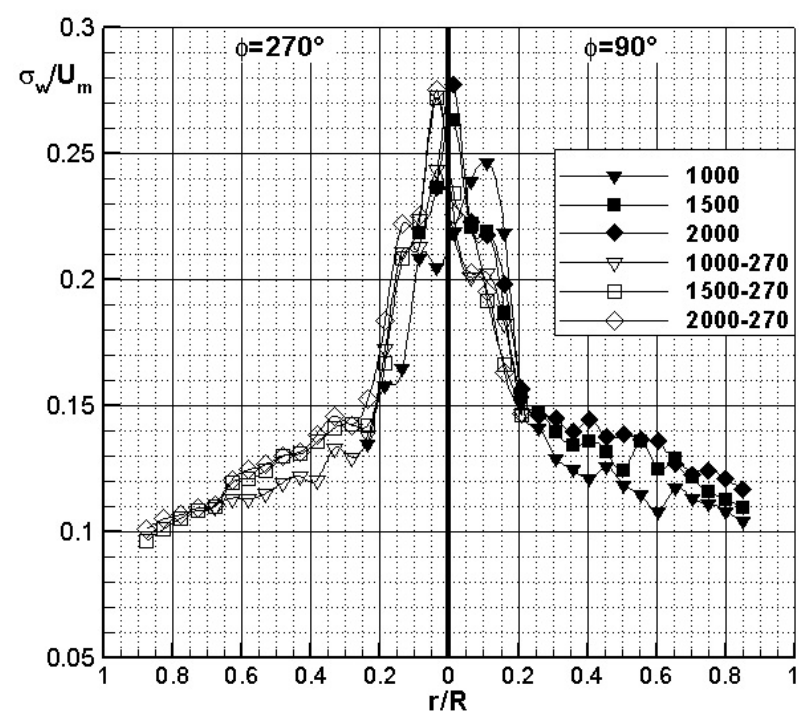

(a)

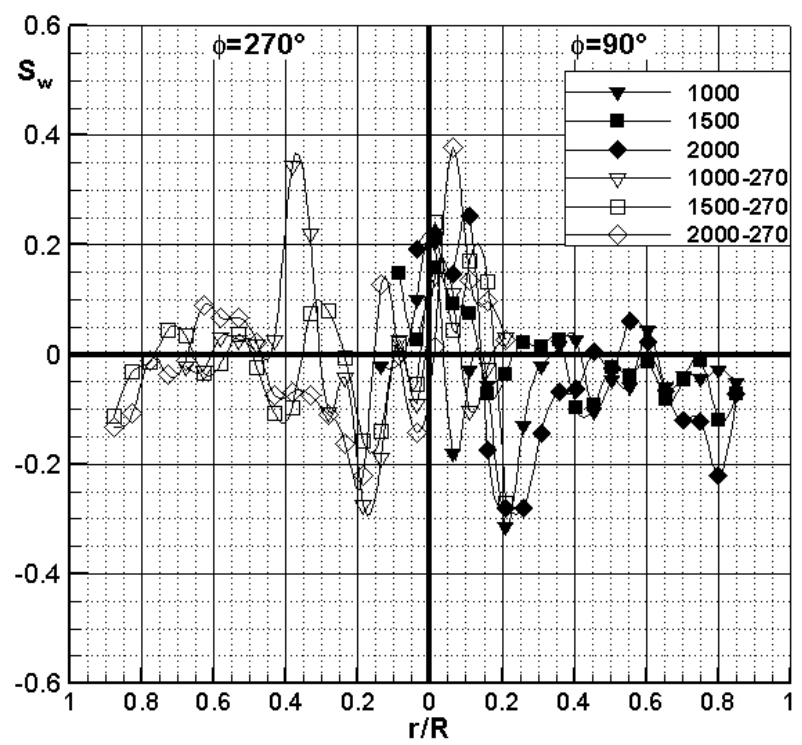

(b)

Fig. 7. Influence of the fan rotation number $(n)$ on statistical parameters for the circumferential velocity in the measuring section $z / D=3.35$ : (a) turbulence level $\left(\sigma_{w} / U_{m}\right)$ and (b) skewness factor $\left(S_{w}\right)$.

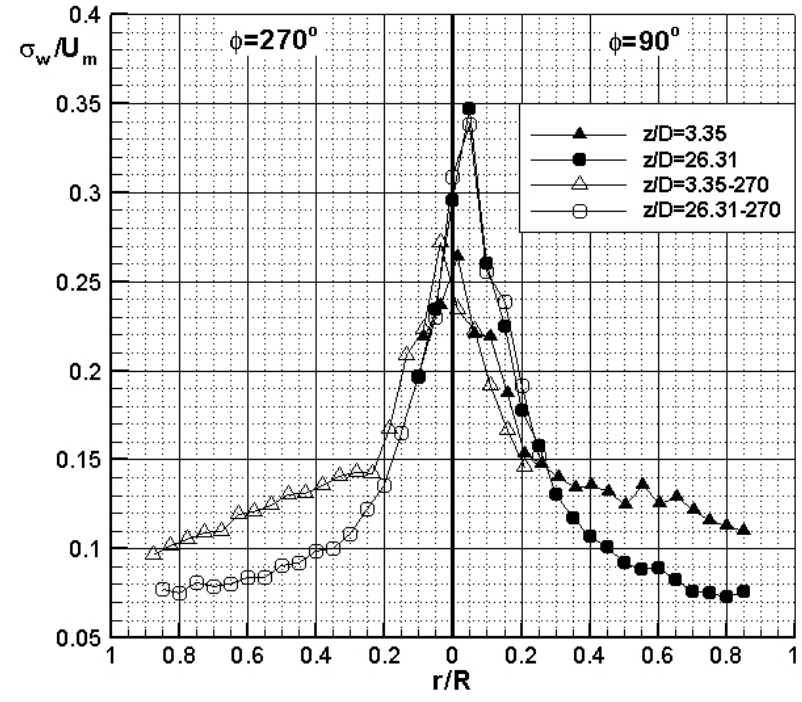

(a)

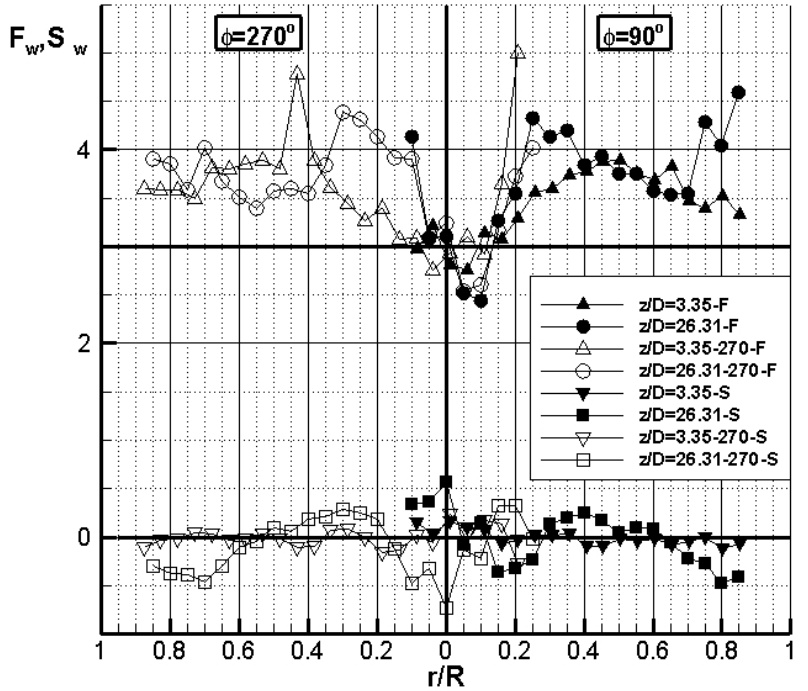

(b)

Fig. 8. Downstream evolution of the statistical moments of the circumferential velocity for regime $n=1500 \mathrm{rpm}$ : (a) turbulence level and (b) skewness and flatness factors.

for its presentation. It is obvious that $\sigma_{w} / U_{m}$ has higher values in the core region in the downstream measuring section, but lower in the sound flow region (Fig. 8a).

Therefore, the turbulence level increases downstream in the vortex core, and decreases in the main flow region. The characteristics of the curves $S_{\mathrm{w}}$ are various, but in principle the values are increased downstream, i.e., negative values become more negative and positive even more positive (Fig. 8b). They differ from the values for normal, Gaussian distribution. The values $F_{w}$ have in the greatest part of the section $z / D=26.31$ higher values than those in $z / D=3.35$, which is important for the domain of vortex core and shear layer. These distributions point out the intermittent character of the generated turbulent swirl flow, as well as the existence of the organized coherent structure in the vortex core and shear layer.

\subsubsection{Autocorrelation function}

The correlation theory incorporates important statements about the structure and statistical nature of 


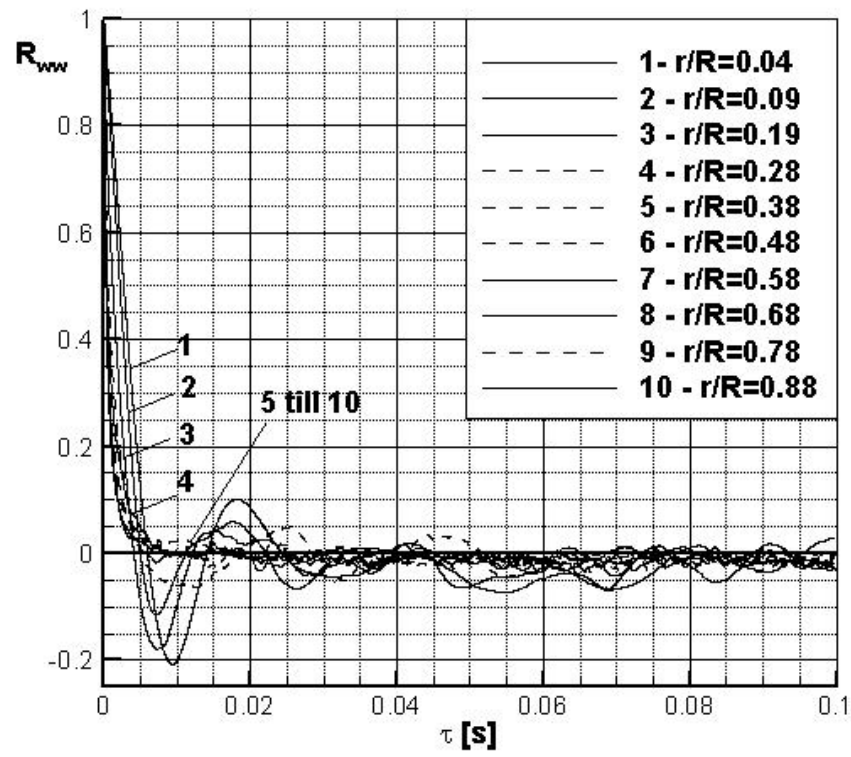

(a)

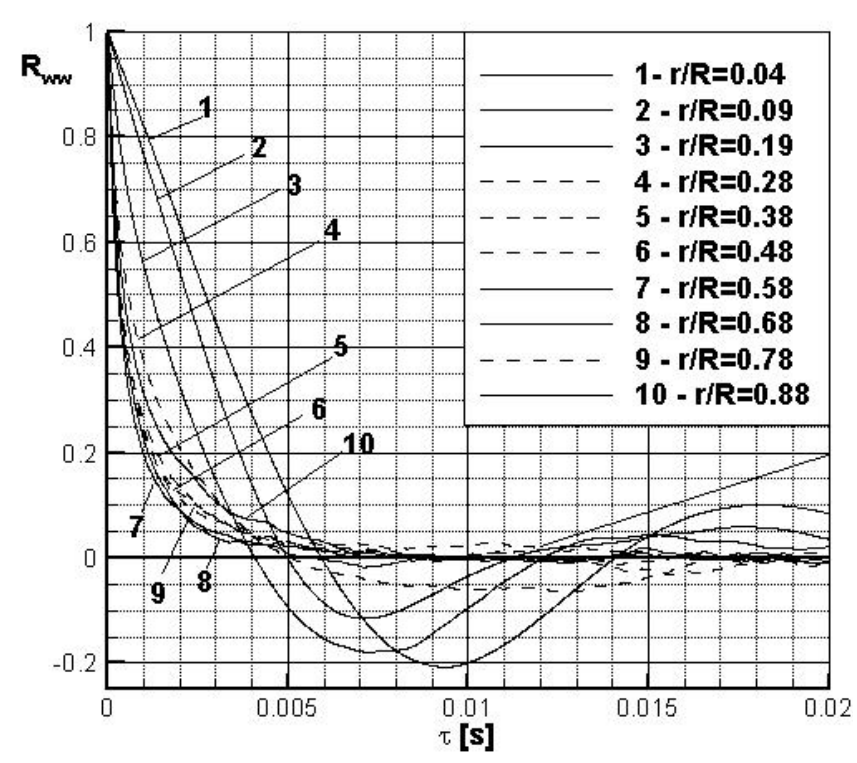

(b)

Fig. 9. Coefficients of time autocorrelation functions for circumferential fluctuating velocity $R_{w w}(\tau)$ in measuring section $z / D=3.35$, plane $\varphi=270^{\circ}$, regime $n=1500 \mathrm{rpm}$, for times: (a) $\tau[\mathrm{s}]=0.1$ and (b) 0.02 .

turbulence. Here, due to the research topic - swirl, it is measured and analyzed the normalized time autocorrelation function for circumferential fluctuating velocity defined as follows:

$$
R_{w w}(\tau)=\overline{w(t) w(t+\tau)} / \sigma_{w}^{2}
$$

which is referred to as the coefficient of time autocorrelation function, or the time autocorrelation coefficient. Distributions of autocorrelation coefficient $R_{w w}(\tau)$ are presented on the basis of measurements in the section $z / D=$ 3.35 , plane $\varphi=270^{\circ}$, for the regime $n=1500 \mathrm{rpm}$, in points which belong to the characteristic flow regions.

Correlation curves are presented for two maximum times $\tau$, to better focus on the existent differences in correlation dependences $\overline{w(t) w(t+\tau)}$ in the regions of vortex core, shear layer and main flow. Namely, the forms of experimentally determined correlation curves indicate various dynamics of fluctuating circumferential velocity fields in measuring points. Sets of correlation curves $R_{w w}(\tau)$ change the sign several times, asymptotically approaching the zero value (Fig. 9). Correlation curves change their positions, depending on the section zone and correlation time $\tau$. In points $r / R=0.58$ and 0.68 there are changes of correlation coefficients significance, i.e., extremely great with $\tau$ increase. It means that high frequency components of circumferential fluctuating velocities play the main role in this region. The character of correlation curves in the core $(r / R=0.04 ; 0.09$ and 0.19$)$ and shear layer $(0.18<r / R<0.38)$ shows the dominant role of low frequency fluctuations (Fig. 9).

Negative values of the correlation function indicate the presence of periodical behaving in the fluctuating circumferential velocity field, when low frequency fluctuations are dominating. In this sense, time $\tau_{0}$, which denotes time interval till the first $R_{w w}\left(\tau_{0}\right)=0$, is related to the dominant frequency of circumferential velocity fluctuations, when the spectral density maximum occurs. Time $\tau_{0}$ can be related to the average frequency of fluctuating circumferential velocities, however, certain analytical relations of these parameters exist only in a strongly expressed periodicity.

\subsection{Stereo PIV measurements}

\subsubsection{Velocity distributions}

Stereo PIV measurements in specified cross-sections provided the distributions of averaged velocity magnitude $\left(c=\left(U^{2}+V^{2}+W^{2}\right)^{0.5}\right)$ on the basis of 400 images (Fig. 10).

Quality measurement area in the measuring section $z / D=3.35$ has maximum dimensions of app. $180 \mathrm{~mm} \times$ $100 \mathrm{~mm}$, while in the second measuring section $200 \mathrm{~mm} \times$ $100 \mathrm{~mm}$. In this way, most of the measuring section is captured in the second case in the $x$-axis direction $r / R \approx 0.5$. The Rankine vortex structure of the turbulent swirl flow is generated by the axial fan and part of it is measured with stereo PIV. In this way, the vortex core and shear layer regions are captured, while in some directions the sound flow region as well. The wall region is not intended to be captured in these measurements.

The maximum velocity achieved in the measuring cross-section $z / D=3.35$ is $c_{\max , 1} \approx 18.4 \mathrm{~m} . \mathrm{s}^{-1}$, while in the second measuring section it is approximately $c_{\max , 2} \approx$ $15 \mathrm{~m} . \mathrm{s}^{-1}$. In both cases it is achieved in the outer regions. Velocity decrease is in correlation with the fact that turbulent swirl flow decays downstream and that velocity field redistribution occurs. The vortex center, defined as 


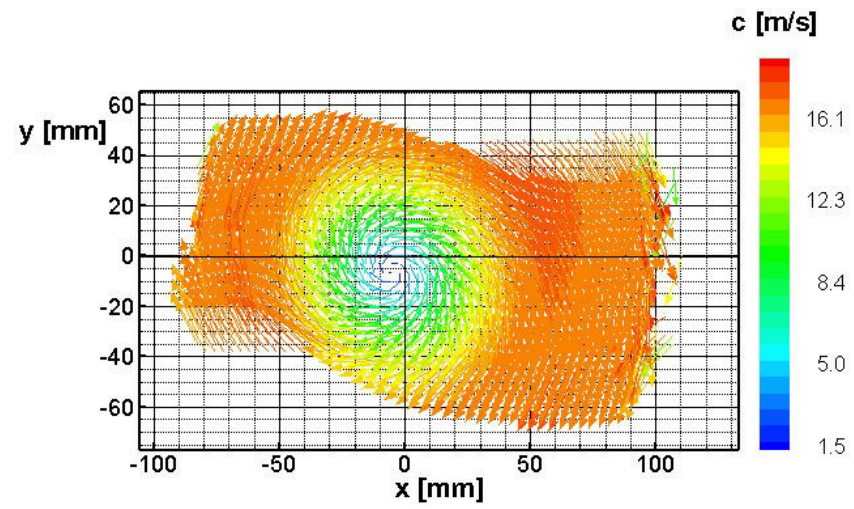

(a)

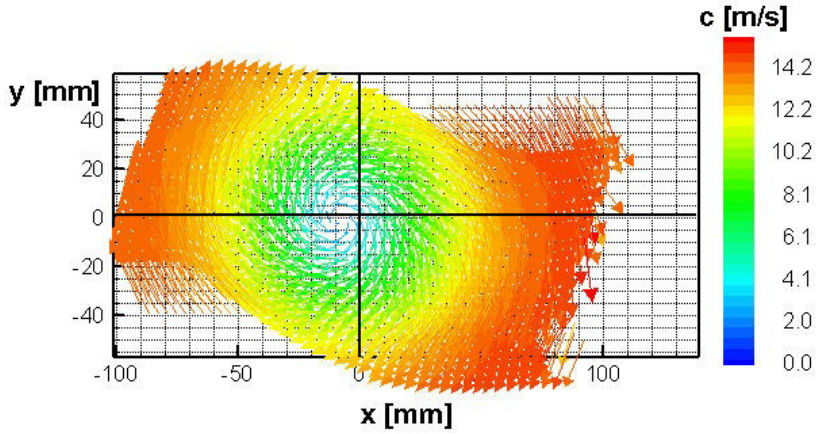

(b)

Fig. 10. Average velocity magnitude distributions in measuring cross-sections for regime $n=1500 \mathrm{rpm}$ : (a) $z / D=3.35$ and (b) $z / D=26.31$.

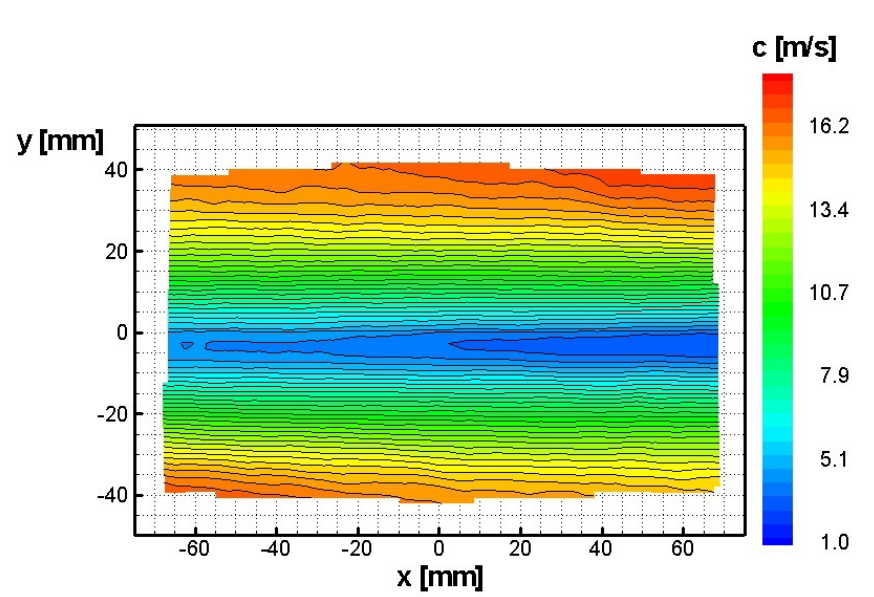

(a)

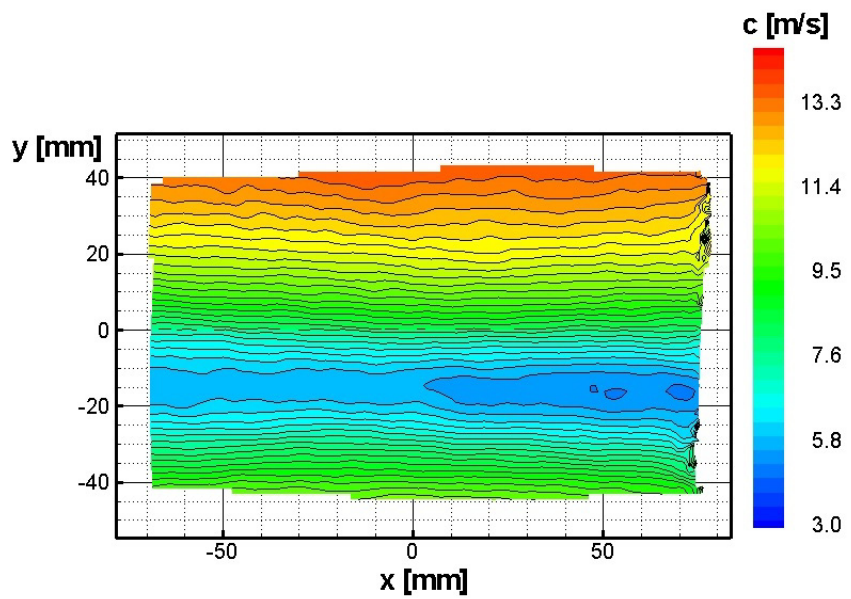

(b)

Fig. 11. Average velocity magnitude distributions in vertical meridian sections for regime $n=1500 \mathrm{rpm}$ : (a) $z / D=3.35$ and (b) $z / D=26.31$.

the velocity magnitude minimum, is not on the pipe axis, nor is it in both cases in the third quadrant. Performed LDA measurements have also shown asymmetrical swirl behavior.

Averaged velocity magnitude of 400 pictures, obtained by stereo PIV measurements with the sampling rate of $2 \mathrm{~Hz}$ in both measuring vertical meridian sections, is presented in Figure 11. Captured area in the section $z / D=3.35$ is app. $135 \mathrm{~mm} \times 80 \mathrm{~mm}$, while in the second section app. $145 \mathrm{~mm} \times 85 \mathrm{~mm}$. In this way, only $r / R \approx 0.36$ is reached, while flow downstream development can be observed. The results of the measurements in the adequate cross- and vertical meridian sections are in correspondence. The Rankine vortex structure is also detected in this plane (Fig. 11).

This correspondence is obvious in the velocity intensity and distribution, as well as in the velocity magnitude minimum positions. The velocity magnitude minimum is about $5 \mathrm{~mm}$ under the pipe axis in the first section (Fig. 11a), while it is higher in the second (Fig. 11b).
These measurements were performed in completely different times, what proves the assumption about the quasistationary flow.

Figure 12 shows the average velocity magnitude distribution in the measuring section $z / D=3.35$, sampled with $7 \mathrm{~Hz}$ and averaged on the basis of only 99 pictures, due to the limitations in RAM. It is obvious that results correspond to those sampled with $2 \mathrm{~Hz}$ in the same measuring section. This again proves the assumption about the quasi-stationary flow.

Distribution of the averaged velocity magnitude preserved a similar character, while magnitudes are slightly different. Distributions of the average axial and circumferential velocities on the basis of 400 pictures in the vertical meridian section with origin in $z / D=3.35$ are presented in Figure 13.

The Rankine vortex structure of the turbulent swirl flow is obvious in the captured regions: vortex core, shear layer and only a part of the sound flow region. Both velocities are symmetrically distributed. However, the vortex 


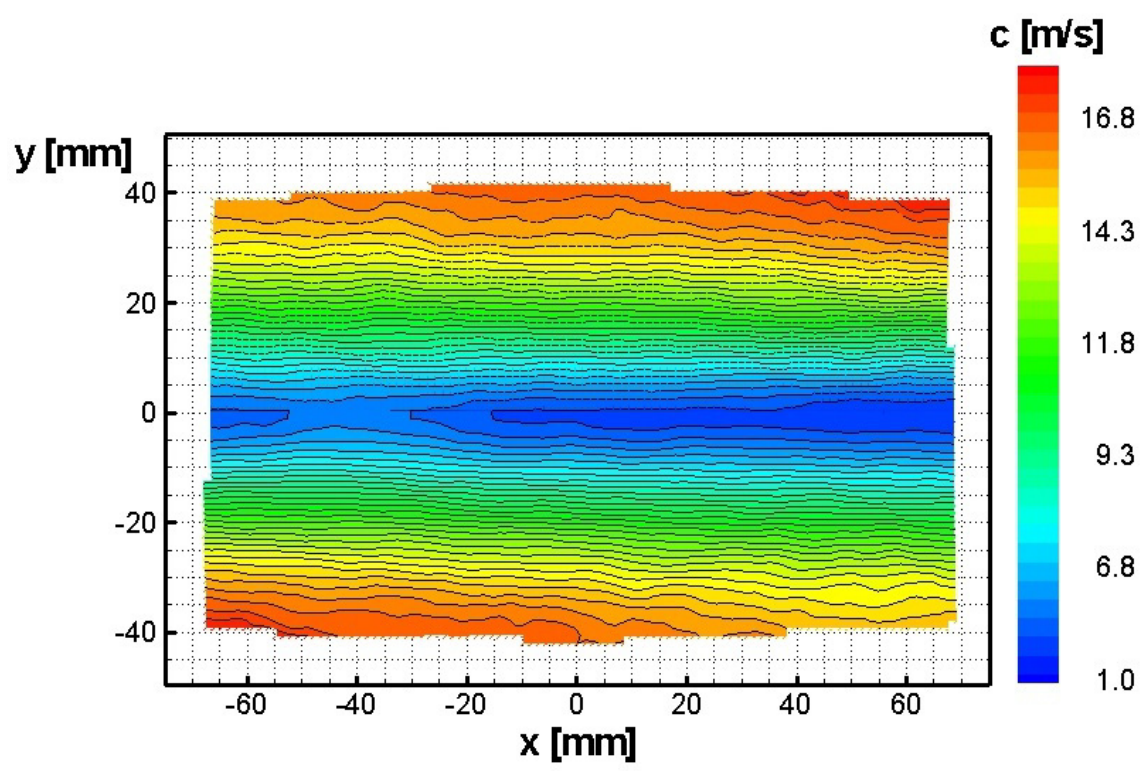

Fig. 12. Average velocity magnitude (c) in the vertical meridian section in origin $z / D=3 / 35$ obtained with $7 \mathrm{~Hz}$ for regime $n=1500 \mathrm{rpm}$.

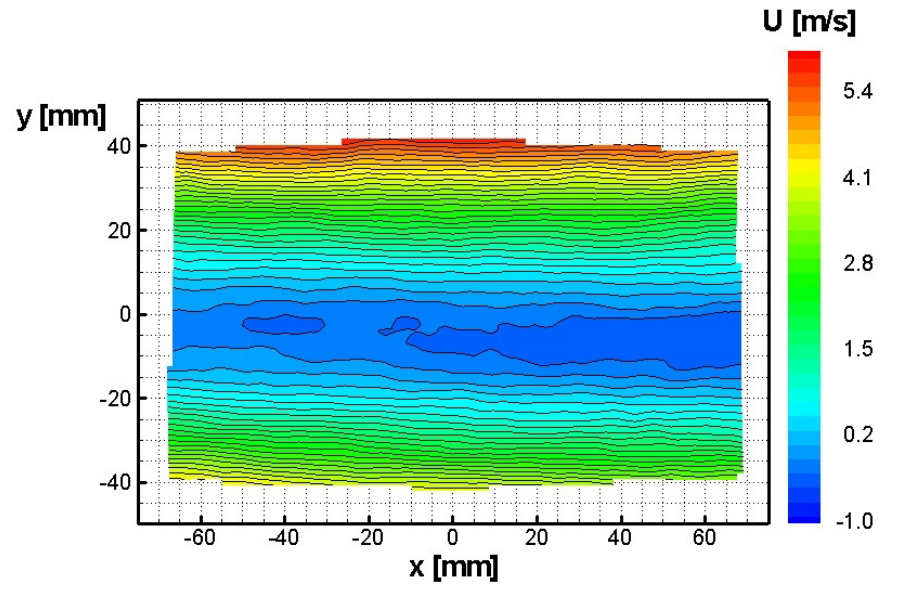

(a)

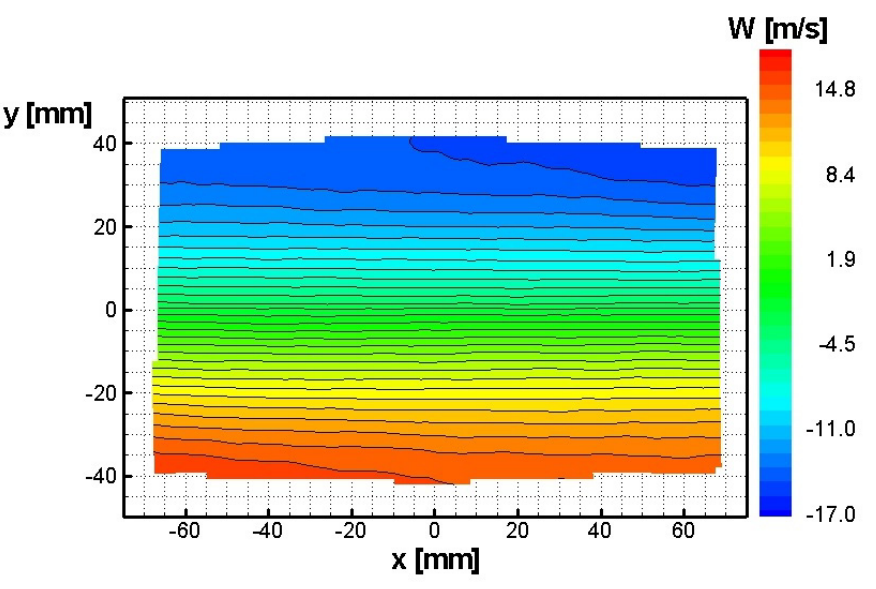

(b)

Fig. 13. Average velocities in the vertical meridian section with origin in $z / D=3.35$ and for regime $n=1500 \mathrm{rpm}$ : (a) axial $(U)$ and (b) circumferential $(W)$.

core center and pipe axis do not overlap, as reported above. Reverse flow is obvious in the vortex core center for the axial velocity in Figure 13a. Circumferential velocity has linear distribution in the vortex core region, which is a characteristic of solid body structure. It reaches its maximum intensities, like axial velocity and velocity magnitude, in the outer regions. Negative values of circumferential velocity originate from the software nomenclature.

All these experimental results and analyses reveal the turbulent swirl flow field, generated behind the axial fan without the guide vanes, durability and complexity. Additional hydraulic elements, such as valves, elbows, diffusers and etc., which is a case in the ventilation systems, can only generate more complex cases and even increase hydraulic losses and power consumption [5]. This implies that turbulent swirl flow should always be considered in designing ventilation systems.

\subsubsection{PIV measuring uncertainty}

The sources of systematic error in the case of the stereo PIV measurements are calibration and data acquisition. Detailed analyses of all errors for the stereo PIV system used in these experiments are presented in reference [17]. For the fan rotation speeds $n=1000,1500$ and $2000 \mathrm{rpm}$, the laser interval between the pulses was $\Delta t=60,40$ and $30 \mu \mathrm{s}$, respectively. "Frozen" particles are omitted in this way. The velocity measuring uncertainty by the use of stereo PIV considering measurement uncertainty of space and time is defined in reference [18]. 


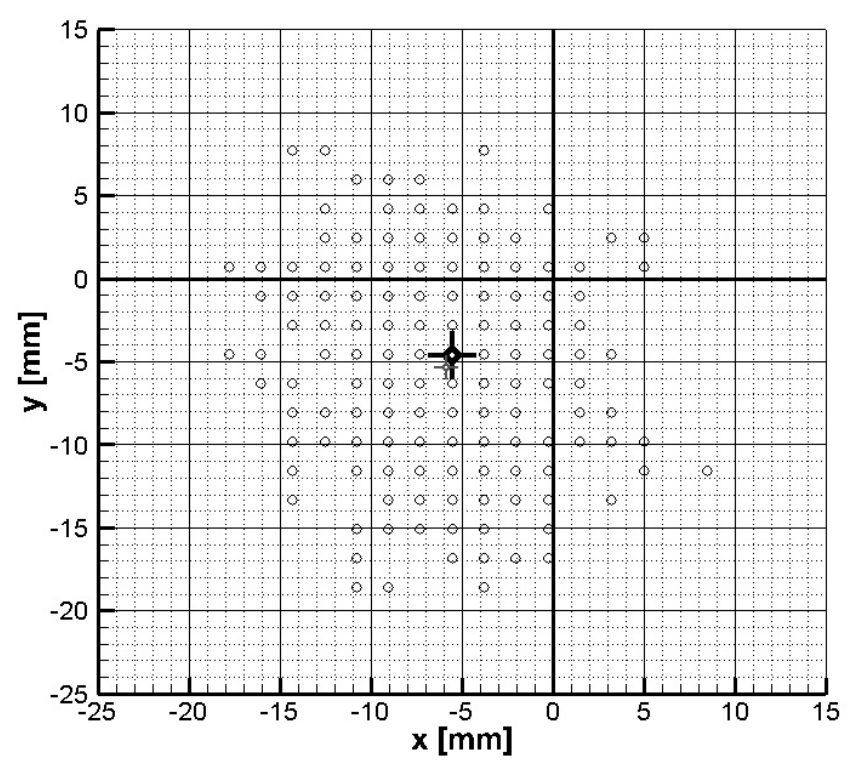

(a)

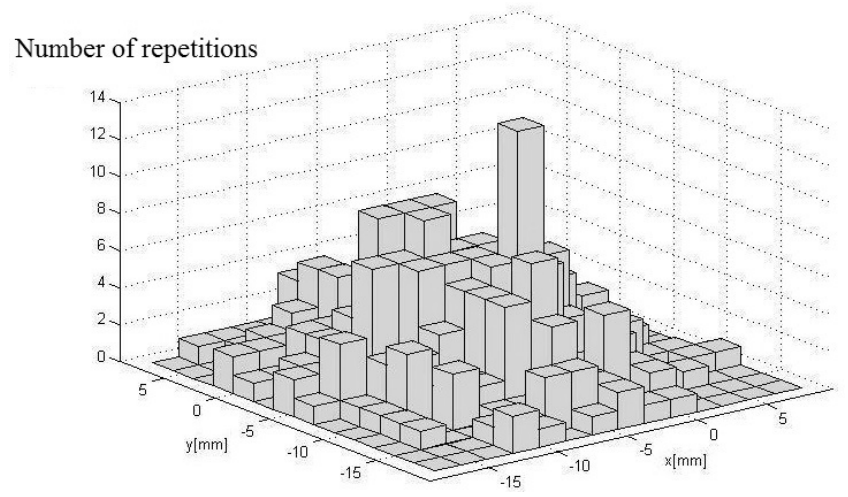

(b)

Fig. 14. Measuring section $z / D=3.35$ and rotation number $n=1500 \mathrm{rpm}$ : (a) positions of the velocity magnitude minimum and (b) histogram of the number of repetitions of the positions of the velocity magnitude minimum in the $(x, y)$ plane.

It seems that the biggest error source is particle move $(\Delta x)$. The rule of five particle pairs inside each interrogation area, omitting pixel blockage, excellent camera focus on particles and $\Delta t$ check aid in decreasing of this error. Used algorithms for data processing are reported in the Section 3.2. Percentage of the interpolated vectors is not higher than $5 \%$. Both cameras result with a small particle move in the vortex region, which is lower than 0.5 pix and higher, app. 4 pix, for other flow regions. Anyhow, it is shown [5] that in this way the error is $1.25 \%$ in the region of shear layer and sound flow region, while it is almost ten times higher in the vortex core region. So, it is almost impossible to measure the whole turbulent swirl flow region at once with equally distributed error.

\subsubsection{Investigation of vortex core dynamics}

Analyses of turbulent velocity field and its structural parameters have shown that characteristic asymmetry of the distribution of measured statistical parameters exists. The vortex core dynamics is directly related to these effects. The principle of the velocity magnitude minimum has been involved as the criterion for vortex core dynamics study. Namely, a set of statistical points with a common property is determined on the basis of measurement results. The common property is the velocity magnitude minimum for at least once. Further statistical-numerical procedure calculates repeatability of the velocity magnitude minimum in the point which is a member of this set of points. Data sampling rate is $2 \mathrm{~Hz}$ for 400 images, i.e., realizations, while $7 \mathrm{~Hz}$ gave 99 images. Positions of the velocity magnitude minima for 400 images acquired with $2 \mathrm{~Hz}$ in the measuring section, are presented in Figure 14a.
The geometry center of all 400 positions is marked with a small cross, while the minimum of the average velocity field is marked with a big black cross. They almost totally overlap and both markers are in the third quadrant. However, all 400 points are not visible, because a great percentage of them is repeated. Only $11.5 \%$ in $z / D=3.35$, while $36 \%$ in the measuring section $z / D=26.31$, are unique. It is clear that the number of unique points is significantly bigger in the downstream section, and therefore the vortex core dynamics is greater in this section. Figure 15b shows a histogram with the number of repetitions in the cross-section $z / D=3.35$, also for data sampling rate of $2 \mathrm{~Hz}$. The histogram provides a number of repetitions of the velocity magnitude, i.e., vortex core center, in each point of the measuring section.

The histogram (Fig. 14b) can also reveal isolines (lines with the same number of repetitions of the position of the velocity magnitude minimum presented in Fig. 15a). Comparison with the isolines in Figure 15b for the same flow regime $(n=1500 \mathrm{rpm})$, but in the measuring section $z / D=26.31$, leads to the conclusion that a more narrow area of the precession movement of the vortex core is downstream. It is obvious that in section $z / D=3.35$ there exist the positions with double the number of maximum repetitions in section $z / D=26.31$.

It is interesting to show that the character remains almost the same and even for higher data sampling rate, but only 99 images (Fig. 16).

The region of turbulent vortex core movement is defined in this way. These developed and applied methods are significant for further statistical investigations of the vortex structure and intermittency of vortex coherent 


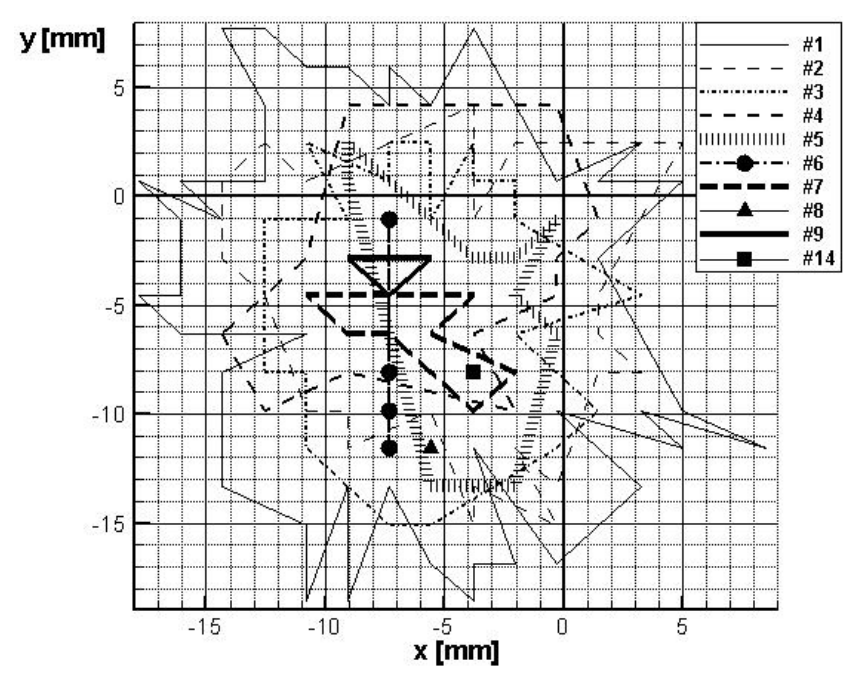

(a)

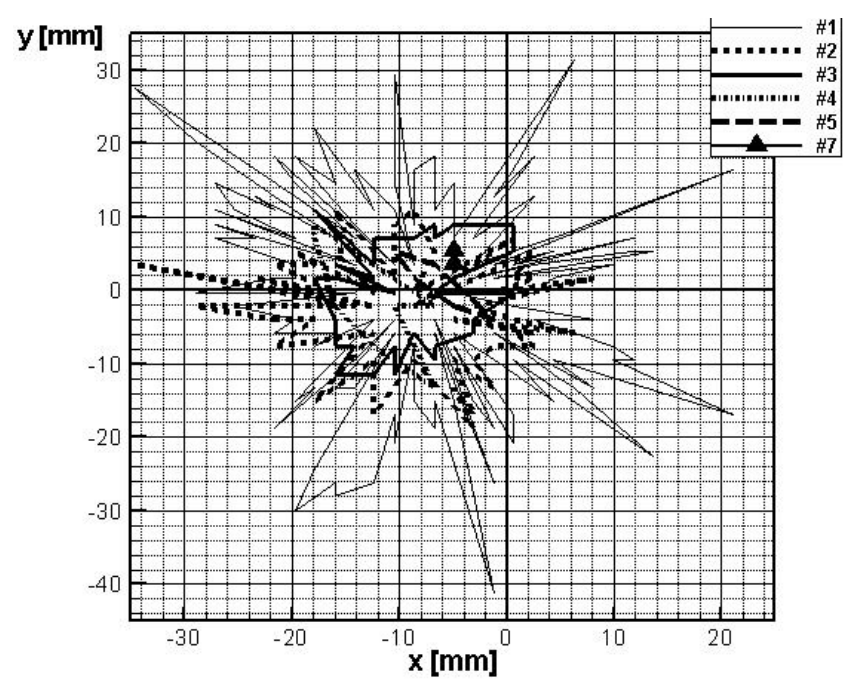

(b)

Fig. 15. Isolines of the repetition number of the velocity magnitude minimum positions for the regime $n=1500 \mathrm{rpm}$ and data sampling rate $2 \mathrm{~Hz}$ in sections: (a) $z / D=3.35$ and (b) $z / D=26.31$.

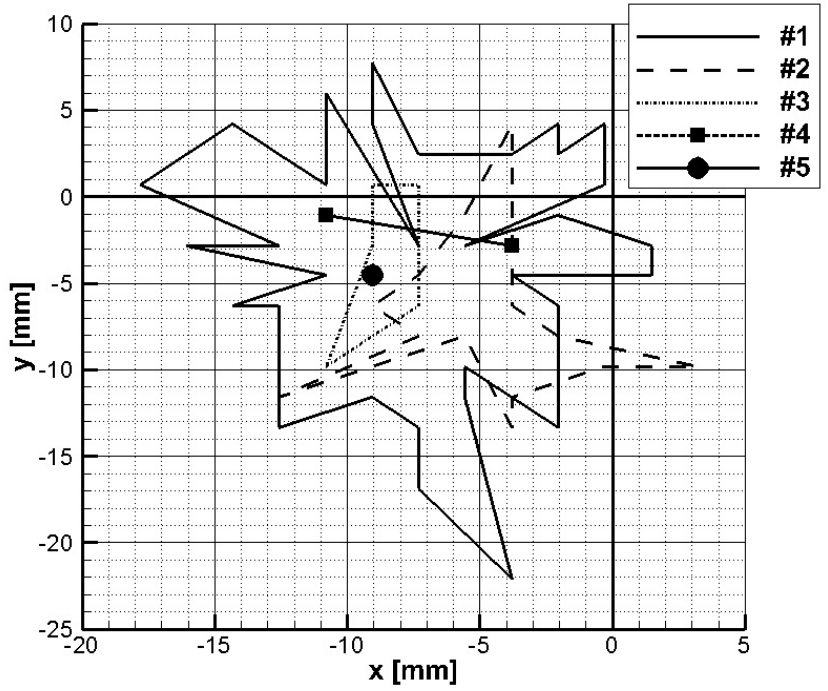

(a)

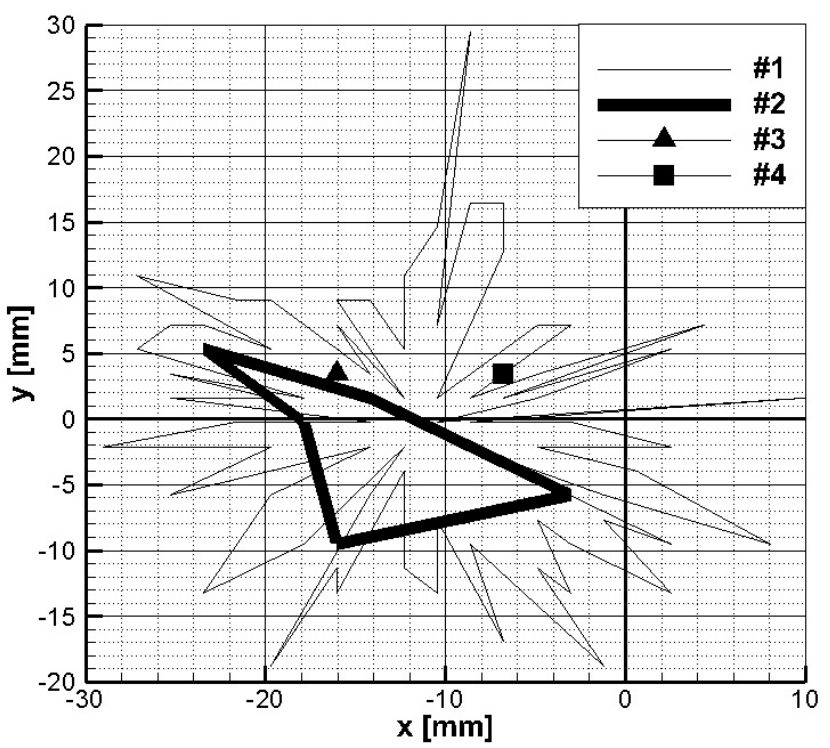

(b)

Fig. 16. Isolines of the repetition number of the velocity magnitude minimum positions for the regime $n=1500 \mathrm{rpm}$ and data sampling rate $7 \mathrm{~Hz}$ in sections: (a) $z / D=3.35$ and (b) $z / D=26.31$.

structures in the core and shear layer of the investigated turbulent swirl flow behind the axial fan impeller.

\section{Conclusions}

Specially designed axial fan impeller was used without guide vanes to generate the turbulent swirl flow in the pipe. One-component LDA and stereo PIV measuring techniques were employed in this study. Characteristic regions: vortex core, shear layer and almost the whole sound flow region were measured. LDA measurements revealed flow integral parameters and turbulence statistics. Non-dimensional velocity profiles show various behaviors. It is shown that obtained axial velocity profiles in measuring section $z / D=3.35$ are similar for various Reynolds numbers. Axial velocity was not transformed downstream almost at all, while circumferential changed the intensity, but not the character. Average circulation in the measuring section is decreased downstream. In both measuring sections radial velocity has the highest values in the vortex core region. 
Turbulence levels reached the highest values in the core region in both measuring sections. They increase downstream in the vortex core, and decrease in the main flow region. Statistical moments of the third and fourth order reveal the intermittent character of the generated turbulent swirl flow and existence of the organized coherent structure in the vortex core and shear layer.

A reverse flow region is generated by the fan, as well as a linear distribution of the circumferential velocity in the vortex core region, which is characteristic of the solid body structure. It is also shown that the average velocity magnitude profile in the downstream section is transformed, but the character of the Rankine vortex remains. Despite the existing vortex core dynamics, the assumption about the quasi-stationary flow is proved by comparing the velocity intensities and distributions in crossand vertical meridian sections, and by applying lower and higher data sampling rates.

Investigation has revealed and proved turbulent swirl flow endurance. This should be considered in designing energy efficient ventilation systems and choosing fans, which are still in most cases built in without guide vanes.

Acknowledgements. Axial fan is designed by Prof. Dr.-Ing. Zoran Protić ${ }^{\dagger}$ (1922-2010) and Prof. Dr. Zoran Stojiljkoviæ designed and built a very precise original fan rotation speed regulator. This research was financially supported by the Ministry of Education, Science and Technological Development, Republic of Serbia, Project No. TR 35046, what is gratefully acknowledged.

\section{References}

[1] K. Yokota, K. Kurahara, D. Kataoka, Y. Tsujimoto, A.J. Acosta, A study of swirling backflow and vortex structure at the inlet of an inducer, JSME Int. J. Ser. B 42 (1999) 451-459

[2] F. Bario, T.M. Faure, E. Jondeau, J.-L. Normand, J.-M. Nguyen Duc, Analysis of inducer recirculating inlet flow, J. Propul. Power 19 (2003) 52-528

[3] W.T.W. Cory, Fans and ventilation: A practical guide, Roles \& Associates Ltd, Elsevier, 2005

[4] M.H. Benišek, S.M. Čantrak, M.S. Nedeljković, Theoretical and experimental investigation of the turbulent swirling flow characteristics in circular pipes, ZAMM 68 (1988) 280-282

[5] Đ.S. Cantrak, Analysis of the vortex core and turbulence structure behind axial fans in a straight pipe using PIV, LDA and HWA methods, Ph. D. thesis (in Serbian), University of Belgrade, Faculty of Mechanical Engineering, Belgrade, 2012
[6] Đ.S. Čantrak, M.S. Nedeljković, N.Z. Janković, Turbulent swirl flow characteristics and vortex core dynamics behind axial fan in a circular pipe, in: Proceedings from Conference on Modelling Fluid Flow (CMFF'12), edited by J. Vad, Budapest, Hungary, 2012 II, pp. 749-756

[7] M.R. Lečć, A.S. Ćoćić, S.M. Čantrak, Original measuring and calibration equipment for investigation of turbulent swirling flow in a circular pipe, Experimental Techniques 38 (2014) 54-62

[8] P. Mattern, S. Sieber, Đ. Čantrak, F. Fröhlig, S. Caglar, M. Gabi, Investigations on the swirl flow caused by an axial fan: A contribution to the revision of ISO 5801, in: Fan 2012, International Conference on Fan Noise, Technology and Numerical Methods, Senlis, France, 2012

[9] J.M.F. Oro, R. Ballesteros-Tajadura, E.B. Marigorta, K.M.A. Díaz, C.S. Morros, Turbulence and secondary flows in an axial flow fan with variable pitch blades, J. Fluids Eng. 130 (2008) 041101.1-041101.11

[10] Z.D. Protić, M.S. Nedeljković, Đ.S. Čantrak, N.Z. Janković, Novel methods for axial fan impeller geometry analysis and experimental investigations of the generated swirl turbulent flow, Thermal Sci. 14 (2010) 125-139

[11] J. Guangyuan, H. Ouayang, D. Zhaohui, Experimental investigation of unsteady flow in axial skewed fans according to flow rates, Exp. Thermal Fluid Sci. 40 (2013) $81-96$

[12] W. Steenbergen, J. Voskamp, The rate of decay of swirl in turbulent pipe flow, Flow Meas. Instrum. 9 (1998) 67-78

[13] J.M. Burazer, M.R. Lečić, S.M. Čantrak, On the nonlocal turbulent transport and non-gradient thermal diffusion phenomena in HVAC systems, FME Trans. 40 (2012) 119-125

[14] S.S. Ristić, J.T. Ilić, D.S. Čantrak, O.R. Ristić, N.Z. Janković, Estimation of laser-Doppler anemometry measuring volume displacement in cylindrical pipe flow, Thermal Sci. 16 (2012) 1127-1142

[15] J.T. Ilić, S.S. Ristić, Đ.S. Čantrak, N.Z. Janković, M. Srećković, The comparison of air flow LDA measurement in simple cylindrical and cylindrical tube with flat external wall, FME Trans. 41 (2013) 333-341

[16] S. Wereley, L. Gui, A correlation-based central difference image correction (CDIC) method and application in a four-roll mill flow PIV measurement, Exp. Fluids 34 (2003) 42-51

[17] J.G. Leishman, M. Ramasamy, Benchmarking PIV with LDV for rotor wake vortex flows, Collection of Technical Papers - AIAA Appl. Aerodyn. Conf. 3 (2006) 1796-1824

[18] R.J. Adrian, Particle-imaging techniques for experimental fluid mechanics, Annu. Rev. Fluid Mech. 23 (1991) 261-304 